\title{
Molecular Regulation in Seed Development Influencing the Fiber Growth in Gossypium hirsutum L.
}

\section{Muhammad Junaid Zaghum 1-3*, Muhammad Nouman Khalid ${ }^{3}$, Muhammad Zia ul Haq ${ }^{2,4}$, Muhammad Tashfeen Gul ${ }^{4}$, Ifrah Amjad ${ }^{3}$ and Muhammad Irfan}

${ }^{1}$ Seed science and technology, Department of plant breeding and genetics, University of Agriculture Faisalabad, Pakistan

${ }^{2}$ Seed physiology Laboratory, Department of Agronomy, University of Agriculture

Faisalabad, Pakistan

${ }^{3}$ Department of plant breeding and genetics, University of Agriculture Faisalabad,

Pakistan

${ }^{4}$ Department of Agronomy, University of Agriculture Faisalabad, Pakistan

*Corresponding Author: Muhammad Junaid Zaghum, Department of plant

breeding and genetics, University of Agriculture Faisalabad, Pakistan.
Received: December 26, 2020

Published: February 15, 2021

(C) All rights are reserved by Muhammad Junaid Zaghum., et al.

\begin{abstract}
Cotton crop is mostly cultivated for fiber production, source of livestock meal and seed oil. The oil of cottonseed used as edible oil around the World after colza and soybean oil and ranked $3^{\text {rd }}$ Worldwide. The quality of cottonseed mainly dependent on protein, oil content, minerals and seed composition. Therefore, However, it is necessary to keeping the acceptable oil content quality. The nutritional value and industrial usage of cottonseed is influences with formation of fatty acids in seed. There is still much unidentified physiological processes and genetic structures that enforce the levels of these metabolites in cottonseed. The expression of homoeologous genes is inappropriate when the epigenetics and intergenomic differences are induced by the genomic association of A and D progenitor in allotetraploid cotton. Signaling factors associated with cell growth, adaptation and development controls the expression of RNA during transcription. Transcriptional factor of MYB-domain encoded by degraded homologous mRNAs during mediation of miRNA that are utilized for fiber development and leaf initiation. From last many decades, scientists work on enhancement of seed and fiber quality. The main nutritional function of cottonseed is oil content and very extensive studies are ongoing on this value-added aspect. Major advancement is taken to increasing the quantitative amount of fatty acid accumulation, which is yet needed to strengthen the requirement and increases the demand of oilseeds. The main objective of this study is to understand the physiologically perspective of seed development and oil content accumulation. To understand the fiber biology to interpret the molecular events which activate or suppress the initiation of fibers from epidermis cells. Because of large genomic size, presence of low-density genetic map and restrictions in cotton transformation.
\end{abstract}

Keywords: Epigenetics; Intergenomic; A and D Progenitor; Cell Growth; MYB-domain; Seed and Fiber Quality; Fatty Acid; Physiological Seed Development

\section{Abbreviations}

DPA: Day Post Anthesis; TRY: Triptychon; CPC: Caprice; SSH: Suppression Subtractive Hybridization; LTP: Lipid Transfer Protein;
PEP: Plastid Membrane-Localized Phosphoenolpyruvate; LKR: Lysine Ketoglutarate Reductase; SDH: Saccharopine Dehydrogenase. 


\section{Introduction}

Cotton is the leading fiber crop and a substantial form of edible oil. Cotton seeds evolve seed coat with cellulose-enriched fibers (lint) and metabolize embryonic proteins and oils. Cottonseed is ultimate form of seed in which either filial and maternal tissue is essential for use [38]. The main biologically useful seed tissue in many crops is filial tissue. Like monocot seeds including rice, wheat and maize primarily develop both protein and starch in endosperm whereas dicotyl seeds such as beans and peas metabolize such products in embryo. For each kg of lint, a cotton plant typically produces around $1.6 \mathrm{~kg}$ of seed. The fuzzy seed is generally used specifically for feed or refined into different products, involving hull, feed, oil and lint. Consequently, cotton is the fifth main oil crop worldwide and the second most valuable source of oil and protein with nearly $21 \%$ and $23 \%$ respectively. Subsequent large output of cotton seeds as by product of fiber for textile industries is sustained by persistent demand for fiber [45]. Based on the key research goal to improve fiber quality and fiber yield, the quality of cotton seed was initially slightly disregarded. This ongoing human preference for high yields and improved fiber content has limited the genetic diversity appropriate for optimizing the quality of cotton seed by breeding [21]. Thus, it is important to provide a precise analysis of physiological occurrences that decide the total compositions of cotton seed storage reserves for maximizing seed production, efficiency, and consequently open the door to major value added by engineering new characteristics [5]. Although this overview focuses mainly on cotton seed development and metabolic activities that enables the seed growth and development.

Cotton fiber development: Synchronized with seed and embryo development

The development of cottonseeds, like that in other angiosperm plants, has its existence in the double-fertilization process which produces triploid cells that develops endospermic tissue [5]. When zygote and endosperm are developed, the maternal seed layer begins to distinguish from the external ovules of the embryo sac throughout. The seed coat is an essential part of the embryo's safety and nutritional transmission from the maternal tissue to all parts of the seeds [44]. The fiber of cottonseed holds the seed from scattering in some wild species. In growing seed coat, internal epidermal cells enlarge steadily with anthesis to generate fibers which are expanding and drying up into fibers and harvested at maturity [5].

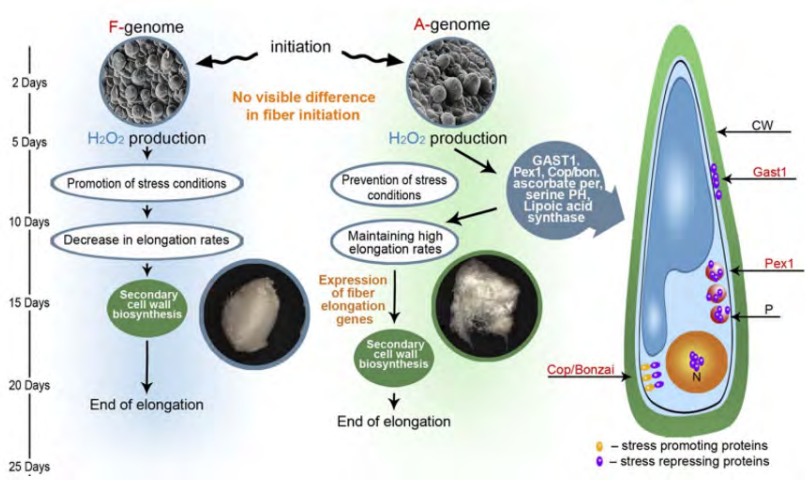

Figure 1: Developmental phases of fiber.

Consequent fiber flexural strength approx. 21 DPA was shown in the wild A- and F-genome of wild species. Our developed design illustrating the physiological and developmental mechanisms which resulted in the initiation of fiber is shown in Figure 1. Initial morphologic evaluation of different growing cotton, particularly most of the F- and A- genome of wild species, shows that for species with short or long fibers, the early phases of fiber initiation and expansion are morphologically comparable [46]. Fiber cells in both the F- and A- genomes at 2 DPA appear as the same symmetrical protrusion. This phase is considered a sign of gradual cell expansion, a process called to engage stimulation of the cell wall, that has been shown to involve $\mathrm{H}_{2} \mathrm{O}_{2}$-mediated non-enzymatic reactivity and certain ROS that form polysaccharides [48]. The oxidation reactions produced $\mathrm{H}_{2} \mathrm{O}_{2}$ enzymatically that is helpful in cell elongation process. The stiffening of cell wall stimulates apparently cell elongation and sometimes its hinder by higher level of $\mathrm{H}_{2} \mathrm{O}_{2}$, and it may be enhancing the chances of necrosis and cell death. Scientist evaluated that sufficient cell elongation effectively occurred by proper levels of ROS. A loss of regulation of cellular $\mathrm{H}_{2} \mathrm{O}_{2}$ and some other ROS levels triggers the shortened developmental period of the F-genome compared to the A-genome, gradually halting elongation and contributing to the development of secondary cell wall formation (Figure 1). In order to differentiate secondary walls in cultivated cotton fibers, $\mathrm{H}_{2} \mathrm{O}_{2}$ was indicated as a progression sign which shows that the prevention of $\mathrm{H}_{2} \mathrm{O}_{2}$ development or the scavenging of internal $\mathrm{H}_{2} \mathrm{O}_{2}$ system inhibits cell wall differentiation [50]. Likewise, the exogeneous $\mathrm{H}_{2} \mathrm{O}_{2}$ addition facilitates 
premature development in young fibers of secondary cell walls. As suggested by expression variations between the F- and A- genomes for the cellulose synthase A1 (CeSA1) gene [18], our findings were consistent with this understanding and with earlier secondary cell wall development in the F-genome (Figure 1). CeSA1 is a gene participating in the synthesis of fiber secondary cell wall and CeSA1 RNA chromatin structure have been proposed as a secondary wall cell synthesis marker. The enhancement of level of $\mathrm{H}_{2} \mathrm{O}_{2}$ exposed during enlargement in A-genome just like in F-genome [46]. However, A-genome fibers did not present elevated RNA levels of stress-related genes, indicating that this lineage formed a mechanism through functional or regulatory processes to regulate ROS levels. The A-genome fibers are upregulated and functional with antioxidants that controlled by genes such as glutathione peroxide, ascorbate peroxide and synthase of lipolic acid. During the further studies by using quantitative RT-PCR found two candidate gene that express short and long fibers. The Cop1/BONZAI (Cop1) gene is a membrane-binding, calcium-dependent protein identified in Arabidopsis from a mutant with a temperature-dependent growth defective and an increased disease tolerance phenotype [49]. Even more research has shown that Cop1 gene serves as a repressor for disease resistance $(R)$ and hence inhibits programmed cell death (PCD) processes [47].

\section{Embryogenesis}

The embryo development is normally categorized in three sequential phases, maturation, desiccation and embryogenesis. The endosperm stretches to the majority of post-fertilization embryonic glands after fertilization and rejuvenates the embryo in growing stage [8]. The funiculus tights composition sustaining the embryo and providing it with nourishment before it gradually diffuses in growth. The cell is initially split after 3-5 days after the anthesis (DPA). The embryo turned in the heart stage takes place at 6-17 DPA. The exclusion and formation of both vascular and embryonic tissues at torpedo stage after 18 DPA. The enlargement of embryo continues till 25 DPA. The symmetric developmental stage is associated with embryo and fiber. During the first 25 DPA, gradual cell expansion begins both in embryo and fiber [11]. Zygotic progression and expansion over global, heart and torpedo stage are clearly described, however due to various varieties and environmental factors, the schedule of the significant activities observed variable in each phase. There are two wide cotyledons in cotton embryos that possess phytin, lipid and storage protein except starch. Although according to other dicotyledonous plants, including legumes, cot- ton cotyledons are minor tightly structured and encased all over the axis of embryo [8]. During 20 days of embryo development (24-40 DPA), the weight and volume of the cotyledons are raised and oils are gathered quickly, and proteins are the key sources of carbon and nitrogen for germination and growth. The fiber retains its thick supplementary cell wall of about cellulose in this phase of embryo development. The forming and abscission of the epidermal cells of the non-fiber seeds usually appears in this stage [11]. The mature cotton seed kernel comprises about $80 \%$ of the dry weight of both proteins and lipids. Dissimilar from many oilseed species, including rapeseed the rapid aggregation of cotton seed proteins correlates with the lipid accumulation [37]. The funiculus continues to dissolve after 45 DPA at ending phase of growing seed and both fiber and seeds are decomposed due to inadequate of a vascular association. The embryo accomplishes its peak dry mass as the amount of water reduces. Resilience and desiccation tolerance are the strengths of embryos at that stage and remain ungerminated [17].

\section{Genes controlling cotton fiber initiation}

Development of one out of 3-4 epidermal cells of ovule into fiber cells is much curious phenomenon in fiber development. To understand the fiber biology the most important step is to interpret the molecular events which activate or suppress the initiation of fibers from epidermis cells [24]. Because of large genomic size, presence of low-density genetic map and restrictions in cotton transformation [8], researchers have moved toward Arabidopsis species with a clear-cut dominance in a map-based cloning and a revolution for seeking potential evidences to regulate cotton fiber initiation. Unicellular trichome advancing from stem or leaves in Arabidopsis is governed by transcriptional factors belonging to R2R3 MYB genomic family [15]. Transcriptional factors WD40, TTG1, GL3 and GL1 function as categorical regulator while CPC and TRY and their enhancers act as negative regulators for the development of trichome and patterning of root hairs. To test the R2-R3 MYBs involvement in cotton fiber development, cDNA library of ovule was screened by the use heterotopous probes evolve from the preserved part of MYB DNA-binding domains and almost six MYB genes were confirmed [16]. Among all these genes, nothing showed fiber-clear-cut expression. Same technique was used to separate 55 more MYB cDNA from ovule of cotton and 61 of the MYB genes were determined in cotton fiber and ovule. Despite of occurrence of excessive MYB genes in cotton, their execution in fiber development remains deceptive of MYB genes that were determined were 
involved in embryo and gamete formation but a gene GhMYB109 was involved in fiber development [20]. Extremely, conversion of trichome less GL1 Arabidopsis mutant with alternative cotton MYB gene GaMYB2, separated from diploid species of Gossypium arboretum, recovered trichome accumulation in the mutant leaves, confirming the fiber MYB gene role in trichome development. Further research results of these authors also suggested that in wild Arabidopsis species the occurrence of this gene under 35S promoter [16], almost 4\% seeds of T1 transgenic plants developed from one hair/seed [28]. Hair formation frequency is negligible and use as an evidence that GaMYB2 can play remarkable role in the development of trichome from seed [16].

GaMYB2 gene expression in the cotton may give a reliable prove of its role in fiber initiation. Some morphological similarities exist in cotton fibers and trichome but there is no clear evidence available which shows that the initiation of cotton fiber is controlled in similar ways as that of trichome. However, some experimental observations determined that various cells types are controlled by numerous molecular mechanisms [25]. There are various spontaneous cotton mutants that exhibit phenotypes of fibreless or fiber reduced [36]. None of those mutations, however, tend to be deficient in production of trichomes. In fact, various loci have been mapped to the cotton genome which is involved in controlling the growth of leaf trichomes and seed fibers. Clearly, more struggles are required to estimate the mechanisms that regulate the initiation of fibers in seed and the production of trichomes from other plant organs. Cotton fibers as sink cells are metabolically active and use sucrose and hexose contents as a metabolites or possible signals upon the ovule epidermis surface for their initiation and protrusion [13]. Many lines of affirmation are homogeneous with this statement or supportive of it. Second, there is about $150 \mathrm{Mm}$ of glucose and fructose contents in the production of cotton fibers [16]. This concentration of hexose is twice of seed coat, estimated at same level and almost 50 times greater than that of the leaf epidermis containing trichome. The high ratio of hexose to sucrose shows the successful metabolism of sucrose and the heavy activity of the sink [39].

\section{Molecular regulation during seed development}

Globally gene expression events are involved in embryogenesis of cotton, especially early events ensuring fertilization, are still broadly unknown, the conclusive genetic influence of production of cotton seed and physiology [8]. Legumes and Arabidopsis are broadly used in various recent years as a model plant for profiling genomic expression and have offered some comprehension into the events that may occur in the cotton [50]. In most angiosperm species, developmental events resulting in creation of mature seeds from single-cell zygotes are absolutely organized and relatively preserve [43]. The first impression of developmental pathways highly involves in the zygotic embryogenesis have been provided by gene regulation found through mutagenesis [37].

In activating/repressing multiple gene sets, various signals transductions pathways are involved and most are identified and characterized by its expression. Therefore, physiological and biochemical processes are determining on seed formation [37]. T- DNA insertion mutagenesis in Arabidopsis revealed a large number of genes necessary for seed production and interestingly categorical in expression pattern. Ultimately, this is necessary for developing essential role in plant life cycle. Although little has yet been done for understanding the genes networking and signaling pathways needed for embryogenesis in cotton and incredible difference observe from model plants. In Arabidopsis, transcriptional factors emphasize the embryogenesis quickly, transduction signal kinase mediated pathway and protein that create and maintain auxin hormone gradient [42]. For instance, home box (WOX) transcription factor genes associated with WUSCHEL label fate decision of cell during early embryogenesis. For cell division that shape domain of the apical embryo, this gene is essential. PIN-formed genes encode transporter-like membrane proteins that are essential for controlling the auxin transportation and the formation of embryonic apical-basal axis is disrupted by PIIN1 and PINI7 mutations [2]. Seed production is epigenetically regulated and for instance, DNA methylation is important for embryogenesis in plant and for viability of seed. Arabidopsis plants with MET1 (METHYLTRANSFERASE1) and CMT3 (CHROMOMETHYLASE3) produce embryos that flourish poorly and have low viability. Genes that determine the identity of embryo cells are mis-expressed and gradient of auxin hormone in abnormal met1 are not appropriately developed [22]. Repression of root-promoting genes cascade in epical embryo domain is a central feature of transition process of embryogenesis, when apical and basal organ consequence is formed [37]. The behavior of associated proteins in which auxin transcriptional mediation are operated together with the AUX/IAA proteins [22].

\section{Somatic embryogenesis}

In cotton, somatic embryos can be argued into in vitro from meristematic cells that pass through globularly, heart shaped, 
torpedo shaped and cotyledonary stages in parallel with developmental pathway of zygotic embryo in seeds [38]. Intrinsically, the zygote is embryogenic, whereas somatic embryogenesis requires embryogenic competence to be induced. Some similar characters between zygotic and somatic embryogenesis have been identified despite these differences. The relative inaccessibility of the young embryo to experimental manipulation is one of the major obstacles to understand in detail the events that rule early embryo development in cotton [4], particularly at early stages of embryogenesis involving only one or few cells. Somatic embryogenesis provides an alternative approach that in some ways circumvents this problem, as large amounts of early embryos can be induced and examined at the molecular or biochemical level at the same time. Gene expression associated with somatic embryogenesis are analyzed using suppression subtractive hybridization ( $\mathrm{SSH}$ ) method on somatic embryogenic cultivar of cotton [45]. The genes encoding transporters are major group of gene sensitive to somatic embryogenesis such as LTP (lipid transfer protein). LTP gene expression is closely linked to the first differential somatic embryo tissue and has a regulatory role in regulating the expansion of cell during embryo development. The processing of post transcriptional and transcription controlling is encoded by protein including zinc Finger-HD homeobox protein [7]. The transcriptional co-activators are responsible for ethylene and nonsensible encoded gene with mediated mRNA limits the protective groups and proteins that are followed this induction of various transporters. Homeodomain genes play crucial regulatory function in all multicellular species in the development and differentiation of patterns [33]. SCR is essential in controlling the division of the endodermis radial and initial tissues patterning in shoot and root under the embryogenesis process [9].

\section{Seed developmental metabolism}

Accumulation of primary nutrients and secondary metabolites required for the germination of seed is a key feature of plant embryos. Cotton seed has different types of proteins and oils which have been tested and assessed as a stable, nutritive and valuable component for animals and human beings [10]. The aggregation of lysigenous gossypol glands comprising aldehydes of terpenoids, especially gossypol that are very harmful to non-ruminant animals and humans, is also marked by cotton seed [27]. Recent efforts to understand the molecular and cellular pathways behind the biogenesis of seed storage compounds and the cloning many genes which are involved in these several processes [12], have played an integral part in the production of cotton seeds with an increased nutritive advantage and improved functional characteristics that improve worth of this byproduct [14].

\section{Carbohydrate regulations}

Cotton fruit (bolls) are very good sinks of carbohydrate. The fruit weight can be increased by 15 percent daily [41]. At early stages of seed development, sucrose produced in leaves through photosynthesis is supplied to the different parts of the seed like seed coat and its endosperm, through the funiculus [29]. Sucrose synthase further cleave the sucrose into UDP glucose and fructose. And hydrolyzed in fructose and glucose by invertase enzyme. Results of several studies indicated that Sus play a dominant role over invertase. Glucose of UDP is further carried to fiber [22]. In these cells, it is used directly in the synthesis of cellulose which is a major component of fiber cell. However certain hexoses are utilized to at least temporarily produce starch in seed coats and endosperm [37].

When starch begins to drop in the seed coat, embryos of the cotton seeds start to starch synthesis at 20 DPA. The embryonic starch keeps decreasing at about 40 DPA and about the same amount of complex sugar appears as galactosidase which further consist of raffinose and stachyose. Carbon import into the seed ceases by about 45 DPA. At this stage non-structural carbohydrates rearrange and this vital metabolic activity further drives maturity processes [32]. Raffinose is produced from galactinol through the transfer of galactose to sucrose, while stachyose is produced by a second galactose addition. Mature cotton seeds comprise of more than $10 \%$ of carbohydrates by weight. synthesize sucrose plays an imperative part in the carbohydrate partitioning of young cotton ovules and that enzymes which synthesize galactoside and sucrose phosphate synthase are essential in the accumulation of carbohydrates in developing cotton seeds [23]. Sus is located in the cellularizing endosperm in early seed development but it was present in embryo not at 10 DPA. Sus activity was noticed $>5$ times than that of vacuolar invertase and $>10$ times that of alkaline invertase in the development of cotton embryos, suggesting a significant part for Sus during early cotton seed development in the degradation of incoming sucrose [22]. A fiber less phenotype led to the suppression of Sus expression in the cotton seed coat, while suppressing the functionality in the maternal tissue caused in retarded and unviable seeds [1].

\section{Synthesis of oil in cottonseed}

One of the main compounds produced and accumulated during the development of cotton seeds is oil. It consists almost exclusively 
of three main fatty acid (FAs) molecules linked to the glycerol backbone [30]. Biosynthesis of FA and TAG assembly are greatly compartmented processes located in various subcellular organelles. Fatty acids are carried to the cytoplasm as acyl-CoA thioesters, and the amount of fatty acids is however converted prior to TAG assembly and deposited in the oil bodies (also called as oleosomes) [34]. when attached to phospholipids on the endoplasmic reticulum (ER) surface. The sugars supplying for lipid production in the cotton seed are initially transferred in endosperm through the seed coat in most plant species, including cotton, and then accumulate by embryo [31]. Fructose, glucose and sucrose are among the sugars. Simple sucrose products produced by sucrose synthesis and invertase are consumed via plastidic glycolytic and cytosolic pathways. Several transporters, like the glucose-6-phosphate translocator, plastid membrane-localized phosphoenolpyruvate (PEP) translocator and triosphate translocator exchange intermediates towards the plastid membrane [26].

Pyruvate and PEP are used as source of carbon for fatty acids biosynthesis in plastids. Malonyl CoA, derived from pyruvate, offers an incremental FA synthesis with a sequence of fatty acid syntheses as a double-carbon donor unit [6]. In recent years, contrasted transcriptome and metabolome of oil that accumulate mesocarp of oil palm (Elaeis guineensis) and sugar that accumulate in the buildup of mesocarp of date palm (Phoenix dactylifera) to justify the causes for the significant disparity in distribution of carbon among these species [40]. Only transcripts of the 18 FA biosynthetic enzymes involved in the conversion of fatty acids to pyruvate were found to be substantially improved by an average 13 times in the palm oil [19]. Pentose, plastidial hexose, phosphoenolpyruvate transporters and triosis phosphate are highly accumulated to improve the carbon flow potential in plastids. Biosynthesis and conversion of fatty acids are most successful regulation of oils collection in palm and possible other plants, rather than TAG assembly. Sucrose and glucose are the primary carbon source for seed production in maternal tissue [39].

\section{Formation of storage protein in cottonseed}

Proteins which are stored in seed are produced by endoplasmic reticulum through amino acids which are utilize by embryo and gained further transamination. Transported through a vesicle mediated pathway into protein storage vacuoles [14]. Two important categories of storage proteins in cotton seed are albumins and globulins that varying in characteristics. These proteins are produced and localized during maturation period of cotton seed. Globulins are categorized in 7S (or a-globulin) and 11/12S legumes based on sedimentation rates of their aggregated forms. Seed protein gene expression is temporarily and spatially regulated in cottonseeds [6]. The diploid genome is encoded by proteins, the mRNA stream for cotton seed formation comprises of $30 \%$ leguminous mRNA in the cotton seed development. As a result, regulation of genes that encode seed storage proteins used to form seed-specific transgene expressions [43].

Proteins which are present in cotton seed has significant amounts of arginine, particularly in comparison to certain legume crops. But lysine and somewhat inadequate isoleucine and sulfur amino acids, methionine and cysteine included, are seriously deficient in cotton seeds relative to other main oils including canola and soybean [30]. Genetic enhancement of amino acid and storage proteins profiles are obviously important for non-ruminant animals or humans to use as a source of protein. In recent years, lysine content has been shown to be increased in plant seed in maize and also cotton [43]. RNAi was specifically, the usage of RNAi to repress the production of $\alpha$-Zein in transgenic corn, subsequently the lysine content doubled (4800 ppm). The enzyme that has multifunction like LKR/SDH (lysine-ketoglutarate reductase/saccharopine dehydrogenase) that induces catabolism, has involved in transgenic corn and has an improved approximately 30 -fold to promote free lysine accumulation [10].

\section{Oleosins}

Oleosins are a member of a family proteins in seed connected with the membrane of oil body. They perform two biochemical functions as protectors to maintain oil bodies in seeds that grow and develop mature, and as a signal to detect fatty acids in sprouts [12]. Oleosins has three regions of amphipathic $\mathrm{N}$ and $\mathrm{C}$ terminal and a closely retained core hydrophobic region that can enter the oil body matrix by means of the phospholipid monolayer. The $\mathrm{N}$ and $\mathrm{C}$ polypeptide terminus are connected with the membrane of phospholipid and situated on the surface of oil body [3]. Two related encoding genes, MatP7 and MatP6, which are 77\% similar, determine during cotton embryogenesis ripening and post-abscission periods. oil bodies are successfully developed and used to purify, replenish, or immobilize recombinant proteins in transgenic oleic plants by leveraging the oleosin's ability to anchor on the surface of oil bodies [34]. The seed storage protein accumulates to the N/Cterminal never change the functional activities of oleosin domains, and fusions remain specifically determine with oil body surface [3]. Although the oil bodies are of low density, they can be extracted 
simply by flotation centrifugation from other cellular components. The required protein that are with the fraction of oil body are extracted effectively without expensive chromatographic measures for protein purification. The desired protein is extracted from the oil body using a specific protease to split through the recombinant protein in a sequence [31].

\section{Conclusion}

The main objectives of cotton fiber genetic studies are to identify fiber cell differentiation and enlargement mechanisms and boost fiber and seed quality. There is need to enhance the higher oil content varieties and maximize the potential of new lines. New advancements aimed at enhancing cotton seed oil's fatty acid composition to meet manufacturers' and industries' requirements. The various agro ecological areas are required to fulfill the cotton production gap that ultimately maximize the oil content requirement. The biotechnological and conventional approaches are utilized to enhance the cottonseed oil production, industrial need and nutritional value. This study reveals the development and regulation of gene that expresses the seed formation and oil contents. Biosynthesis of FA and TAG assembly are greatly compartmented processes located in various subcellular organelles. Fatty acids are carried to the cytoplasm as acyl-CoA thioesters, internal epidermal cells enlarge steadily with anthesis to generate fibers which are expanding and drying up into fibers and harvested at maturity, Transcriptional factors WD40, TTG1, GL3 and GL1 function as categorical regulator while CPC and TRY and their enhancers act as negative regulators for the development of trichome and patterning of root hairs and fiber growth that are the highly revealed mechanisms and regulation processes. The mutations and genetic variations are stabilizing by conventional approaches to enhance the oil content in cottonseeds. The cotton is vital source of valuable fiber production and enhancing the potential of by products such as biofuel, feed and food. In this study we evaluate the fiber development and healthy seed formation and emphasize the accumulation of oil bodies which ultimately influences the seed morphogenesis during the physiological and biochemical developmental activities.

\section{Conflict of Interest}

There is no conflict of interest.

\section{Bibliography}

1. Ahmad N., et al. "Heterosis Studies of Yield and Fiber Quality Traits in (Gossypium Hirsutum L .)". The International Journal of Biological Research 2 (2019): 162-74.
2. Anderson OD., et al. "Structural and Transcriptional Analysis of Plant Genes Encoding the Bifunctional Lysine Ketoglutarate Reductase Saccharopine Dehydrogenase Enzyme". BMC Plant Biology 10 (2010) 22-30.

3. Antos JM., et al. "Site-Specific N- and C-Terminal Labeling of a Single Polypeptide Using Sortases of Different Specificity". Journal of the American Chemical Society 131.31 (2009): 10800-10801.

4. Baskaran P and Johannes VS. "Plant Regeneration via Somatic Embryogenesis in Drimia Robusta". Plant Cell, Tissue and Organ Culture 119.2 (2014): 281-288.

5. Berger F., et al. "Endosperm: An Integrator of Seed Growth and Development”. Current Opinion in Plant Biology 9.6 (2006): 664-670.

6. Carbonell-Verdu A., et al. "Development of Environmentally Friendly Composite Matrices from Epoxidized Cottonseed Oil". European Polymer Journal 63 (2015): 1-10.

7. Chew W., et al. "Role of Homeodomain Leucine Zipper (HDZip) Iv Transcription Factors in Plant Development and Plant Protection from Deleterious Environmental Factors". International Journal of Molecular Sciences 14.4 (2013): 8122-8147.

8. Chugh A and Khurana P. "Gene Expression during SE - Recent Advances”. Current Science 83 (2002): 715-730.

9. Cui H., et al. "An Evolutionarily Conserved Mechanism Delimiting SHR Movement Defines a Single Layer of Endodermis in Plants". Science 316.5823 (2007): 421-425.

10. Dowd MK., et al. "Fatty Acid Profiles of Cottonseed Genotypes from the National Cotton Variety Trials". Journal of Cotton Science 14.2 (2010): 64-73.

11. Dumas, C and Mogensen, HL. "Gametes and Fertilization: Maize as a Model System for Experimental Embryogenesis in Flowering Plants". The Plant Cell 5 (1993): 1337-1348.

12. Gai YP., et al. "Chilling Stress Accelerates Degradation of Seed Storage Protein and Photosynthetic Protein during Cotton Seed Germination". Journal of Agronomy and Crop Science 194.4 (2008): 278-288.

13. Guo K., et al. "Fibre Elongation Requires Normal Redox Homeostasis Modulated by Cytosolic Ascorbate Peroxidase in 
Cotton (Gossypium Hirsutum)". Journal of Experimental Botany 67.11 (2016): 3289-3301.

14. He Z., et al. "Protein Profiling of Water and Alkali Soluble Cottonseed Protein Isolates". Scientific Reports 8.1 (2018): 1-9.

15. Hsu CY., et al. "Transcriptional Regulation of the Lipid Transfer Protein Gene LTP3 in Cotton Fibers by a Novel MYB Protein". Plant Science 168.1 (2005): 167-181.

16. Hu H., et al. "GhJAZ2 Negatively Regulates Cotton Fiber Initiation by Interacting with the R2R3-MYB Transcription Factor GhMYB25-Like". Plant Journal 88.6 (2016): 921-935.

17. Hu W., et al. "The Variability of Cottonseed Yield under Different Potassium Levels Is Associated with the Changed Oil Metabolism in Embryo". Field Crops Research 224.1 (2018): 80-90.

18. Huang Y., et al. "Steam Explosion Distinctively Enhances Biomass Enzymatic Saccharification of Cotton Stalks by Largely Reducing Cellulose Polymerization Degree in G. Barbadense and G. Hirsutum". Bioresource Technology 181 (2015): 224230 .

19. Ibrahim AH., et al. "The Effect of Natural and Artificial Fruit Dehiscence on Floss Properties, Seed Germination and Protein Expression in Calotropis Procera". Acta Physiologiae Plantarum 38.1 (2016): 1-11.

20. Jin X., et al. "Using Genome-Referenced Expressed Sequence Tag Assembly to Analyze the Origin and Expression Patterns of Gossypium Hirsutum Transcripts". Journal of Integrative Plant Biology 55.7 (2018): 576-585.

21. Kelly CM., et al. "Genetic Improvement in Cotton Fiber Elongation Can Impact Yarn Quality". Industrial Crops and Products 129.1 (2018) 1-9.

22. Kleczkowski LA., et al. "Update on Sucrose Synthesis/Metabolism UDP-Glucose Pyrophosphorylase . An Old Protein with New Tricks 1". Plant Physiology 134.1 (2004): 912-18.

23. Kundu A and Markkandan G. "GhMATE1 Expression Regulates Aluminum Tolerance of Cotton and Overexpression of GhMATE1 Enhances Acid Soil Tolerance of Arabidopsis". Current Plant Biology 24.1 (2020): 100-160.

24. Lee JJ., et al. "Gene Expression Changes and Early Events in Cotton Fibre Development". Annals of Botany 100.7 (2007): 1391-401.
25. Liu B., et al. "The R3-MYB Gene GhCPC Negatively Regulates Cotton Fiber Elongation". PLOS ONE 10.2 (2015): 1-17.

26. Liu J, et al. "Changes of Sucrose Metabolism in Leaf Subtending to Cotton Boll under Cool Temperature Due to Late Planting". Field Crops Research 144 (2013): 200-11.

27. Liu Q., et al. "High-Stearic and High-Oleic Cottonseed Oils Produced by Hairpin RNA-Mediated Post-Transcriptional Gene Silencing". Plant Physiology 129.4 (2002): 1732-43.

28. Liu YD., et al. "Improved Salt Tolerance and Delayed Leaf Senescence in Transgenic Cotton Expressing the Agrobacterium IPT Gene". Biologia Plantarum 56.2 (2012): 237-46.

29. Magwanga RO., et al. "Characterization of the Late Embryogenesis Abundant (LEA) Proteins Family and Their Role in Drought Stress Tolerance in Upland Cotton". BMC Genetics 19.1 (2018): 1-31.

30. Muntz K., et al. "Stored Proteinases and the Initiation of Storage Protein Mobilization in Seeds during Germination and Seedling Growth". Journal of Experimental Botany 52.1 (2001): 1741-1752.

31. Nikiforidis CV. "Structure and Functions of Oleosomes (Oil Bodies)". Advances in Colloid and Interface Science 274.1 (2019): 102039.

32. Peterbauer T and Richter A. "Biochemistry and Physiology of Raffinose Family Oligosaccharides and Galactosyl Cyclitols in Seeds". Seed Science Research 11.3 (2001): 185-97.

33. Qin YF., et al. "Three Cotton Homeobox Genes Are Preferentially Expressed during Early Seedling Development and in Response to Phytohormone Signaling". Plant Cell Reports 29.10 (2010): 1147-56.

34. Qin YM., et al. "Saturated Very-Long-Chain Fatty Acids Promote Cotton Fiber and Arabidopsis Cell Elongation by Activating Ethylene Biosynthesis". Plant Cell 19.1 (2009): 3692-704,

35. Radhamony RN., et al. "T-DNA Insertional Mutagenesis in Arabidopsis: A Tool for Functional Genomics". Electronic Journal of Biotechnology 8.1 (2005): 82-106.

36. Rehman S., et al. "Effect of Topping under Different Nitrogen Levels on Agronomic Characters of Cotton". The International Journal of Biological Research 2 (2019): 233-245. 
37. Ruan YL., et al. "Expression of Sucrose Synthase in the Developing Endosperm Is Essential for Early Seed Development in Cotton". Functional Plant Biology 35.5 (2008): 382-393.

38. Sawan ZM. "Cottonseed Yield and Its Quality as Affected by Mineral Nutrients and Plant Growth Retardants". Cogent Biology 2.1 (2016): 1080-1093.

39. Saxena DK., et al. "Comparative Extraction of Cottonseed Oil". ARPN Journal of Engineering and Applied Sciences 6.1 (2011): 84-89.

40. Tang Z., et al. "Quantitative Metabolome and Transcriptome Analysis Reveals Complex Regulatory Pathway Underlying Photoinduced Fiber Color Formation in Cotton". Gene 767.1 (2020): 145180.

41. Tung SA., et al. "Mepiquat Chloride Effects on Potassium Acquisition and Functional Leaf Physiology as Well as Lint Yield in Highly Dense Late-Sown Cotton". Industrial Crops and Products 129.1 (2019): 142-155.

42. Wang YH and Hong WY. "How Effective Is T-DNA Insertional Mutagenesis in Arabidopsis?" Journal of Biochemical Technology 1.1 (2008): 11-20.

43. Xie F and Baohong Z. "MicroRNA Evolution and Expression Analysis in Polyploidized Cotton Genome". Plant Biotechnology Journal 13.3 (2015): 421-434.

44. Yan H., et al. "Analysis of the Chemical Composition of Cotton Seed Coat by Fourier-Transform Infrared (FT-IR) Microspectroscopy". Cellulose 16 (2009): 1099-107.

45. Zheng M., et al. "Protein Expression Changes during Cotton Fiber Elongation in Response to Low Temperature Stress". Journal of Plant Physiology 169.4 (2012): 399-409.

46. Applequist W., et al. "Comparative development of fiber in wild and cultivated cotton". Evolution and Development 3.1 (2001): 3-17.

47. Jambunathan N., et al. "A humidity-sensitive Arabidopsis copine mutant exhibits precocious cell death and increased disease resistance". Plant Cell 13.10 (2001): 2225-2240.

48. Potikha T., et al. "The involvement of hydrogen peroxide in the differentiation secondary walls in cotton fibers". Plant Physiology 119.3 (1999): 849-858.
49. Yang S., et al. "The BON/CPN gene family represses cell death and promotes cell growth in Arabidopsis". Plant Journal 45.2 (2006): 166-179.

50. Hovav R., et al. "The evolution of spinnable cotton fiber entailed prolonged development and a novel metabolism". PLoS Genetics 4.2 (2008): 1-9.

\section{Assets from publication with us}

- Prompt Acknowledgement after receiving the article

- Thorough Double blinded peer review

- Rapid Publication

- Issue of Publication Certificate

- High visibility of your Published work

Website: www.actascientific.com/

Submit Article: www.actascientific.com/submission.php

Email us: editor@actascientific.com

Contact us: +919182824667 